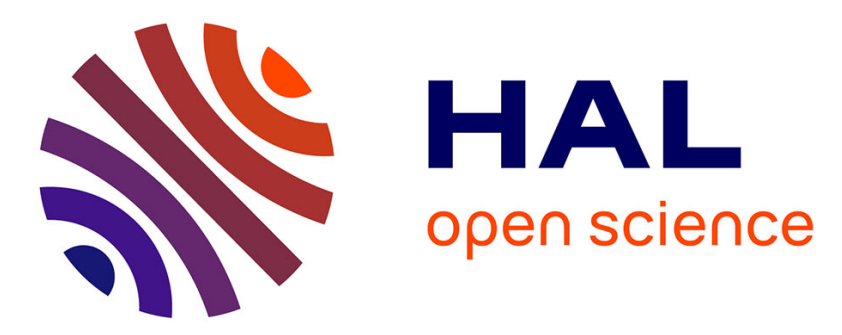

\title{
A Quality Based Approach for the Analysis and Design of Business Process Models
}

\author{
Sarah Ayad
}

\section{To cite this version:}

Sarah Ayad. A Quality Based Approach for the Analysis and Design of Business Process Models. Sixth International Conference on Research Challenges in Information Science, May 16-18 2012, Valencia, Spain, May 2012, Valencia, Spain. pp.1-5. hal-00764078

\section{HAL Id: hal-00764078 \\ https://hal.science/hal-00764078}

Submitted on 12 Dec 2012

HAL is a multi-disciplinary open access archive for the deposit and dissemination of scientific research documents, whether they are published or not. The documents may come from teaching and research institutions in France or abroad, or from public or private research centers.
L'archive ouverte pluridisciplinaire HAL, est destinée au dépôt et à la diffusion de documents scientifiques de niveau recherche, publiés ou non, émanant des établissements d'enseignement et de recherche français ou étrangers, des laboratoires publics ou privés. 


\section{A Quality Based Approach for the Analysis and Design of Business Process Models}

\author{
Sarah Ayad ${ }^{1}$ \\ CEDRIC-CNAM, 292 Rue Saint Martin \\ F-75141 Paris Cedex 03, France \\ sarah.ayad@auditeur.cnam.fr
}

\author{
Supervisors: \\ Isabelle Comyn-Wattiau \\ Samira Si-saïd Cherfi
}

\begin{abstract}
The scientific problem addressed in this paper consists in modeling and improving Business Process (BP) models quality. This problem is of growing interest as companies are realizing the undeniable impact of a better understanding of business processes (BP) on the effectiveness, consistency and transparency of their business operations. The research aims at proposing methods and tools for BP model quality measurement and improvement. We propose a semantic approach of quality exploiting domain knowledge.
\end{abstract}

Keywords-component: Business Process Models, Quality metrics, Quality Measurement, Quality Improvement

\section{INTRODUCTION TO RESEARCH TOPIC}

In recent years, the problems related to modeling and improving business processes has been of growing interest. Indeed, companies are realizing the undeniable impact of a better understanding and management of business processes (BP) on the effectiveness, consistency and transparency of their business operations. BP modeling aims at a better understanding of processes, allowing deciders to improve them. We propose to support this modeling effort with a qualitybased approach combining both quality measurement and domain knowledge. The domain knowledge is structured using ontologies. This domain knowledge enables the definition of semantic quality metrics. It is also used during the quality improvement.

\section{SignificANT PRoblems IN THE FIELD AND THEIR CURRENT SOLUTIONS}

In order to make process models understandable, reliable and reusable there is a need to insure their quality. Several approaches to reach such an objective have been proposed. We have identified mainly three categories of contributions: quality-based method, process model quality measurement, and process quality measurement.
We intend by quality-based methods all the approaches providing modeling guides and best practices in order to improve the quality of produced models. In [8] the authors provide guidelines to improve BP models characteristics such as correctness, understandability etc. In [12] the authors discussed the importance of documentation, activity labeling and icons for BP models understanding and acceptance. Other authors proposed a pattern based approach for quality assurance [22].

The second category refers to quality considerations at the process level. This is related to process simulation and process monitoring. [17] presents a set of simulation tools and their evaluation. The authors in [21] discussed several techniques for process verification, validation, and performance improvement.

Our research focuses on quality measurement. Most of the articles proposing quality metrics for business process models mention the similarities between a software process and a business process. Basing on this hypothesis, they apply software metrics to evaluate business process models. [2] defines a set of metrics for the evaluation of BP conceptual models. These metrics provide the quantitative basis necessary to assess the maintainability of the process models. The potential of quality metrics in business process management is studied in [10, 11]. [13] describes a framework enabling the evaluation and comparison of enterprise models based on a syntactic, semantic, and pragmatic analysis. [20] investigate the importance of structuredness for process model correctness from an empirical perspective. They introduce the definitions of two metrics which quantify the structuredness degree of a process model and the unmatched connector count. [9] describes a quality metric permitting to analyze the complexity of workflow patterns from a log-based perspective. [1] proposes a comprehensive state-of-the-art on how existing software complexity metrics were adopted in order to analyze the current BP models complexity and also to propose a goalquestion-metric (GQM) framework for defining these metrics. 
Finally, [7] introduces the cross-connectivity metric that measures how tightly the nodes in a process model are connected. They used their metrics in explaining the variation of understanding and occurrence of errors in process models.

In order to achieve a generic and flexible environment for software measurement, some authors propose a meta-model for the definition of software measurement models. A flexible method to measure any kind of software entity represented by its associated meta-model is proposed by [4]. In [18], authors discuss existing quality models before proposing a meta-model for specifying quality models in the context of model-driven engineering.

Arguing on the lack of a sound consensus on the same concepts and vocabulary in different approaches, several authors present a semiformal ontology for software metrics and indicators, based as much as possible on the concepts of standards [16]. This can be useful to support different assurance processes, methods and tools in addition to be the foundation for a cataloguing web system.

As a conclusion, business process model quality is a very active research topic. However, to the best of our knowledge, there is no framework allowing modelers to semantically improve their models guided by a quality approach. Our contribution is a step forward in this direction. In this paper, we propose to use a domain ontology to improve $\mathrm{BP}$ model semantic quality".

\section{OUR APPROACH AND ITS EXPECTED CONTRIBUTIONS}

In reply to the above evidences, we propose the following steps as our research roadmap:

- First, there exists several business process model notations and we aim to propose an approach that is notation independent. To reach this objective, we first started by defining a meta-model for business process models. This is a result of federating existing work on business process modeling (see Section A for details).

- As we have to reason about domain ontologies, we also defined a meta-model for ontology concepts taking into account the needs of our quality approach (see Section A).

- Our objective is to propose a detailed approach supporting business process modeling with quality based guides. An overall view of the approach is proposed in Section B.

- To validate the feasibility of the approach it will be implemented through a prototype including an interface with main commercial business process modeling tools.

- Finally we plan to validate the quality metrics, the quality improvement guide and the approach by the means of surveys and empirical validation.

\section{A. Definition of meta-models}

Our approach relies on two categories of knowledge. The first one is contained in the process models for which we provide quality evaluation method. The second one represents the domain. Itis considered in our approach as a source for both quality evaluation and improvement. To do so, we need to reason in general about both process models and domain knowledge represented in our approach by domain ontologies. The first result of the thesis work is the construction of a process model meta-model and an ontology meta-model.

An extract from the two meta-models is sketched in Figures 1 and 2. These meta-models are the result of a federation effort of existing literature. A business process model is composed of flow objects, artifacts and connecting elements. A flow object could be a gateway, an event or an activity [15]. An activity can be atomic (task) or non atomic (process). A task is defined as a minimal traceable work that can not be broken down.A connecting element could be an association, a sequence or a message flow. Activities require resources that can be information or things [5]. Thing could be physical, corresponding to physical concepts or peopleResource representing human beings responsible of activity performance or owning the process, etc.

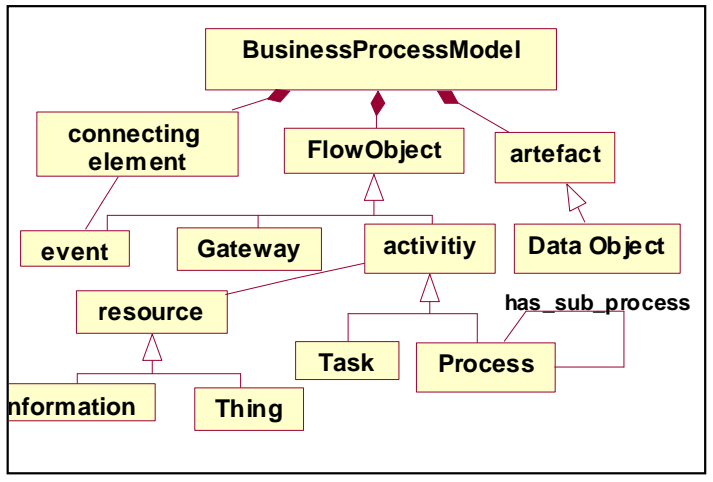

Figure 1. Business process meta-model

An ontology is composed of classes and relationships. For the class definition, we use [19] classification of entities into actor, action and artefact. The relationships are based on a classification adapted from [6]. We consider three categories of relationships namely:

- Status relationships describe durable states induced by events. They correspond to structural relationships such as IS-A, part of, member, etc.

- Interaction relationships capture communications among objects.

- Change in status relationships describe the transition life cycles. Examples from this category are intention to do an action or attempt to perform an action, etc. 


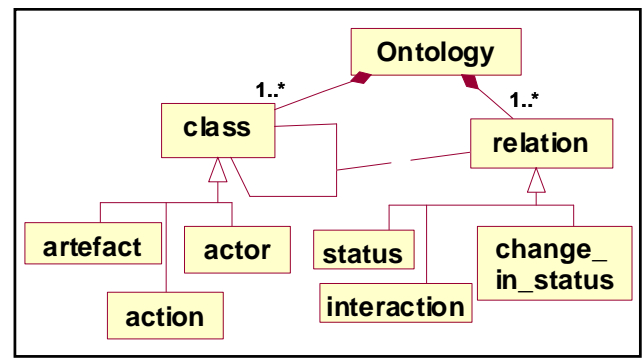

Figure 2. Ontology process meta-model

\section{B. The overall quality approach}

Modeling activity in general and business processes modeling in particular area creative activity conducted by a modeler with the help of a given notation or modeling language. The result is of course highly dependent on the modeler expertise in the notation practice, on his/her interpretation of the reality, and on the decision he/she makes regarding the choice of concepts and details to be modeled. However, this model is supposed to be a faithful representation of the reality. Thus the definition of quality requirements for these models is a mean to evaluate this modeling activity and to ensure a better result. Many quality factors may be defined to characterize this quality. In our approach, we propose to measure the semantic quality of BP models. The semantic quality is related to both the expressiveness of the models and to the ease with which they could be understood regarding the requirements or the domain.

The approach relies on the process having as input point the business process model to be evaluated and a domain ontology representing business knowledge and rules of the underlying problem domain. The steps of the process are the following:

- .Discovering similarities between input BP model and domain ontology: this is based on a set of alignment rules at both syntactic and semantic levels. Syntactic alignment uses a set of predefined alignment rules such as the fact that an 'actor' from an ontology is to be compared to a 'people resource' from the BP model. At the semantics level we use similarity measures based on synonymy, homonymy, etc. relationships.

- Evaluating semantic quality includes measuring a value of quality according to quality metrics. Most of the proposals in the literature concentrate on structural quality. We have started defining some semantic quality metrics such as semantic clarity and semantic completeness.

- Improving semantic quality: An originality of our work is to integrate the quality improvement within the proposed approach. Again for this step, we plan to use the knowledge provided by the domain ontology to improve the model quality according to the results of quality evaluation.

For both similarity discovery and improvement steps, we have started expressing the rules in a formal language.
In order to evaluate the semantic quality, many quality attributes could be identified. We started working on the expressiveness of models. A model is said to be expressive when it represents user's requirements in a natural way [23]. We first identified a set of what we call expressiveness defects. These defects correspond to modeling choices that could decrease the expressiveness of models leading to errors in their interpretation and further their implementation.

Ambiguity defect: aims to avoid interpretation errors. Ambiguity occurs when several modeling elements encountered in the model are identified as being synonyms according to the ontology.

Abstraction defect: aims to use the suitable level of generality. Indeed, in some cases, using general concepts instead of specific and precise ones can decrease the efficiency of the processes. On the contrary, using very specialized terms may decrease the understandability of the models. The relevant choice of an abstraction level depends on several factors among which we can mention the nature of audience (developers or users), the objective of the model (explanation or implementation), etc.

Incompleteness defect: occurs when a concept is complex and only a subset of its components is represented in the model. The partial coverage of the components could be related to an incomplete description of the application domain.

The set of defined defects permits to suspect some quality problems that need to be confirmed by the quality expert/analyst. For example, according to the domain ontology, and considering the model fragment provided, we could suspect an abstraction defect regarding the terms "medical team". The usage of a very general term instead of specific terms such as "nurse", "emergency practitioner" or "cardiologist" could decrease the efficiency of the modeled process.

These defects are used by very simple quality metrics in the current version. They only compute the number of suspected defects for each category.

Here are two examples of metrics to measure the quality of models according to the identified defects:

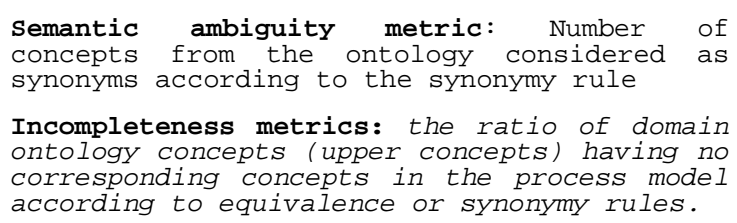

The detection of defects relies on discovering similarities between elements of the process model and concepts of the domain ontology. As an illustration, we describe two examples of OCL synonymy functions. The synonym_a_p function verifies whether a resource of type people (from the process model) has a synonymy closeness with a class of type actor from the domain ontology. The synonymy closeness here is computed by comparing the existence of common names and/or common synonyms using Wordnet. 


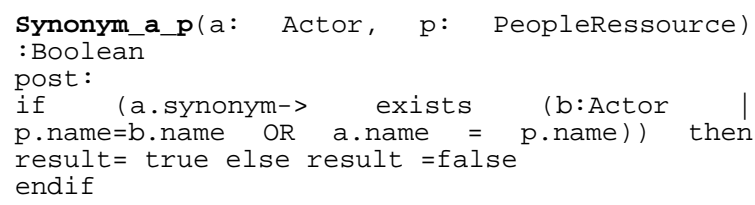

The second function synonyms_p returns a set of concepts (of type actor) from the ontology considered as synonyms of a concept (of type people resource)

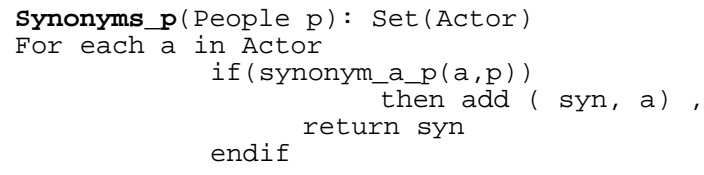

Once the defects detected and the value of quality computed, our approach proposes a set of guides to improve the quality of the BP models.

The quality improvement activity provides the analyst or the quality expert with a set of improvement guidelines to correct the defects. Here are some examples of quality improvement guides:

Correcting ambiguity defects: consists in replacing the confirmed similarities in the model by a unique concept name. Once again, the ontology helps by providing the list of synonyms. The analyst only has to choose among them the suitable term.

Correcting abstraction defects: according to the situation in hand, concepts could be replaced by a more generic one if more generality is needed. However, if more detailed descriptions are needed, the general concept in the model is replaced by more specific ones as we can see in the following example. In both situations there could be an impact on other parts of the model when merging or refining processes for example.

Correcting incompleteness defects: In case of incompleteness, the analyst could rely on the knowledge provided by the ontology to fill the missing parts of the model. For example, if a missing resource is detected this should lead to the addition of this resource and the completion of the process using this resource.

\section{An Automated Environment to Implement our Approach}

We propose to design and develop a prototype implementing the proposed approach. This implementation has two core objectives. It will first help in demonstrating the feasibility of the approach. The second objective is related to the validation of the approach as we plan to make the prototype available to students, researchers and practitioners to collect their feedbacks.

\section{Validation of the Proposed Approach}

The proposed approach will be validated on the basis of the feedback received from different populations (researchers, professionals, students etc.) who have either used the proposed utility for evaluating and improving the quality of their models or have either been interviewed or surveyed over the efficacy of the proposed quality concepts and approach.
Other suitable methodologies can also be used or employed for validation.

- Quantitative Methods; such as surveys to gather data from the respondents. .

- Qualitative Methods such as action research, use case study etc.

\section{E. Further work}

The work presented in this paper is a step forward in semantics based quality evaluation and improvement using domain ontologies. Further research includes the enrichment of quality defects rules and the development of a metrics suite for semantic quality evaluation. We plan also to develop a mean to improve the guidance provided during the improvement activity.

[1] A. Ghani , G.M. Wei , Muketha and W.P. Wen , "Complexity Metrics for Measuring the Understandability and Maintainability of business process Models using goal-question-Metric (GQM)" in: International journal of computer science and network security, Vol. $8 \mathrm{~N}^{\circ} 5$, p. 219225, May 2008

[2] E. Rolon , R. Garcia and M. Piattini, " Applying Software metrics to evaluate Business Process Models" in CLEIEl Ectronic Journal, volume 9, number 1 , paper 5, june 2006

[3] F. Garcia, M. Bertoa, C. Calero, A. Vallecillo, F. Ruiz, M. Piattini and M. Genero "Towards a consistent terminology for software measurement" in: Information and Software Technology Volume 48, Issue 8, August 2006, Pages 631-644

[4] F. Garcia ,M.Serrano , Cruz-Lemus, Ruiz and M. Piattini "Managing software process measurement: A metamodel-based approach" in: Information Sciences Volume 177, Issue 12, 15 June 2007, Pages 25702586

[5] H.E . Eriksson and M. Penker, "Business Modeling With UML: Business Patterns at Work" John Wiley \& Sons, Inc. New York, NY, USA (C2000 ISBN:0471295515

[6] I. Vanderfeesten , J. Cardoso, J. Mendling, Reijers and Alast " Quality Metrics for Business Process Models" in: Fischer, L. (ed.) BPM and Workflow Handbook 2007 (May 2007), pp. 179-190. Key: citeulike: 5757678

[7] I. Vanderfeesten , Reijers, J. Mendling, Aalst and Cardos " On a quest for good Process Models: The Cross-Connectivity Metric" in: Advanced Information Systems Engineering (20th International Conference, CAiSE'08, Montpellier, France, June 18-20, 2008, Proceedings) / Ed. Z. Bellahsène, M. Léonard. - Berlin : Springer, 2008. - ISBN 978-3-54069533-2. - (Lecture Notes in Computer Science ; 5074). - p. 480-494

[8] J. Becker, M. Rosemann, C. von Uthmann " Guidelines of Business Process Modeling" in Business Process Management 2000: 30-49

[9] [9] J. Cardoso "Business Process quality Metrics: Log-based Complexity of Workflow Patterns" in: OTM'07 Proceedings of the 2007 OTM Confederated international conference on On the move to meaningful internet systems: CoopIS, DOA, ODBASE, GADA, and IS - Volume Part

[10] J. Cardoso , J. Mendling, G. Neumann and H.A. Reijers "A Discourse on Complexity of Process Models" in Business Process Management Workshops 2006: 117-128

[11] J. Mendling, H.A. Rejers and J. Cardoso "What Makes Process Models Understandable?" in Lecture Notes in Computer Science, 2007, Volume 4714/2007, 48-63, DOI: 10.1007/978-3-540-75183-0_4

[12] J. Mendling, J. Recker and H.A. Reijers " On the Usage of Labels and Icons in Business Process Modeling" in IJISMD 1(2): 40-58 (2010)

[13] J.P. Van Belle " A proposed framework for the analysis and evaluation of business models" in Proceedings of the 2004 annual research conference of the South African institute of computer scientists and information technologists on IT research in developing countries 
[14] J. Sarkis and S. Talluri" A Synergistic Framework for Evaluating Business Process Improvements" in International Journal of Flexible Manufacturing Systems, Volume 14, Number 1, 53-71, DOI: 10.1023/A:1013827026600, 2002

[15] L. Loja,V. Neto , S. Costa and J. Oliveira "A business process metamodel for enterprise information systems automatic generation" in Brazilian Workshop on Model-Driven development. Brazil 2010.

[16] M. de Los Angeles and L. Olsina" Towards and Ontology for software metrics and indicators as the foundation for a cataloging web system" in: Lecture Notes in Computer Science, 2004, Volume 3140/2004, 778, DOI: 10.1007/978-3-540-27834-4_23

[17] M.H. Jansen-Vullers and M.Netjes "Business Process Simulation A Tool Survey" in Workshop and Tutorial on Practical Use of Coloured Petri Nets and the CPN

[18] P. Mohagheghi, V.Dehlen " A metamodel for specifying quality models in Model-driven engineering" in: Journal Nordic Journal of Computing archive Volume 14 Issue 4, December 2008
[19] S.Purao and V.Storey " A multi-layered ontology for comparing relationship semantics in conceptual models of databases" Appl. Ontol. 1, 1 (January 2005), 117-139.

[20] V. Gruhn and R. Laue "Complexity metrics for business process models" in 9th international conference on business information systems (BIS 2006), volume 85 of Lecture Notes in Informatics

[21] W. M. P. van der Aalst " Challenges in Business Process Analysis" in ICEIS (Selected Papers) 2007: 27-42

[22] W.M.P. van der Aalst, W.M.P, A.H.M. ter Hofstede, B. Kiepuszewski, and A.P. Barros. Workflow Patterns. Distributed and Parallel Databases, 14(3), pages 5-51, July 2003.

[23] C. Batini, S. Ceri, S.B. Navathe, Conceptual database design : An entity relationsip approach, Benjamen Cummings, Redwood City, California, 1992 